\title{
Thoracic radiotherapy plus Durvalumab in elderly and/or frail NSCLC stage III patients unfit for chemotherapy - employing optimized (hypofractionated) radiotherapy to foster durvalumab efficacy: study protocol of the TRADE-hypo trial
}

Farastuk Bozorgmehr ${ }^{1,2^{*}}$ D, Inn Chung ${ }^{1,2}$, Petros Christopoulos ${ }^{1,2}$, Johannes Krisam³ ${ }^{3}$, Marc A. Schneider ${ }^{2,4}$, Lena Brückner ${ }^{1,2}$, Daniel Wilhelm Mueller ${ }^{5}$, Michael Thomas ${ }^{1,2}$ and Stefan Rieken ${ }^{6}$

\begin{abstract}
Background: Non-small cell lung cancer is the most common cause of cancer death worldwide, highlighting the need for novel therapeutic concepts. In particular, there is still a lack of treatment strategies for the group of elderly and frail patients, who are frequently not capable of receiving standard therapy regimens. Despite comprising the majority of lung cancer patients, this group is underrepresented in clinical trials. This applies also to elderly and frail patients suffering from unresectable stage III NSCLC, who are unfit for chemotherapy, and, therefore, cannot receive the standard therapy comprising of radiochemotherapy and the recently approved subsequent durvalumab consolidation therapy. These patients often receive radiotherapy only, which raises the concern of undertreatment. The TRADE-hypo trial aims at optimizing treatment of this patient group by combining radiotherapy with concomitant durvalumab administration, thereby employing the immune-promoting effects of radiotherapy, and determining safety, feasibility, and efficacy of this treatment.

Methods/ design: In this prospective phase II clinical trial, durvalumab therapy will be combined with either conventionally fractionated (CON-group) or hypofractionated (HYPO-group) thoracic radiotherapy. A safety stop-and-go lead-in phase will assess safety of hypofractionated radiotherapy with respect to severe pneumonitis in small patient cohorts before opening full enrollment. Tumor tissue, blood and stool samples will be collected before and during the study period to investigate the immunological mechanisms responsible for checkpoint inhibitor efficacy and immune-promoting effects of radiotherapy.

\footnotetext{
* Correspondence: Farastuk.Bozorgmehr@med.uni-heidelberg.de

${ }^{1}$ Department of Thoracic Oncology, Thoraxklinik at University Hospital of Heidelberg, Röntgenstraße 1, 69126 Heidelberg, Germany

${ }^{2}$ Translational Lung Research Center Heidelberg TLRCH, Member of the

German Center for Lung Research DZL, Im Neuenheimer Feld 156, 69120

Heidelberg, Germany

Full list of author information is available at the end of the article
}

(c) The Author(s). 2020 Open Access This article is licensed under a Creative Commons Attribution 4.0 International License, which permits use, sharing, adaptation, distribution and reproduction in any medium or format, as long as you give appropriate credit to the original author(s) and the source, provide a link to the Creative Commons licence, and indicate if changes were made. The images or other third party material in this article are included in the article's Creative Commons licence, unless indicated otherwise in a credit line to the material. If material is not included in the article's Creative Commons licence and your intended use is not permitted by statutory regulation or exceeds the permitted use, you will need to obtain permission directly from the copyright holder. To view a copy of this licence, visit http://creativecommons.org/licenses/by/4.0/ The Creative Commons Public Domain Dedication waiver (http://creativecommons.org/publicdomain/zero/1.0/) applies to the data made available in this article, unless otherwise stated in a credit line to the data. 
(Continued from previous page)

Discussion: Preclinical data suggests that irradiation-induced immunogenicity can be further increased if applied in a hypofractionated setting, potentially boosting the expected synergistic effect with immune checkpoint inhibition in restoring the immune anti-tumor response. If proven safe and efficient, a hypofractionated radiation schedule can provide a considerably more practicable option for the patient. Taking into consideration the intend to develop a combination treatment strategy that can be made available to patients soon after proving to be efficient and the potentially elevated toxicity of a hypofractionated radiotherapy approach, this trial was designed as a two-trials-in-one design. An accompanying translational research program is planned striving to gain insights into the tumor-host biology and to identify suitable biomarkers to predict therapy response.

Trial registration: Clinicaltrials.gov, NCT04351256. Registered 17 April 2020, Eudra-CT, 2019-002192-33. Registered 24 October 2019,

Keywords: Non-small cell lung cancer, NSCLC, Radioimmunotherapy, Immune checkpoint inhibition, Anti-PD-L1 monoclonal antibody, Hypofractionated radiation, Geriatric risk profile

\section{Background}

Lung cancer is the most common cause of cancer death worldwide, with non-small cell lung cancer (NSCLC) representing $80-90 \%$ of cases $[1,2]$. Improving therapeutic strategies is thus of imminent importance, especially considering elderly and frail patients. With a median age of about 70 years at diagnosis lung cancer clearly is a disease of the elderly, yet this group is underrepresented in clinical trials, and these patients are frequently not capable of receiving standard treatment protocols due to ageand tobacco-associated comorbidities [3-5].

In recent years, the advent of immunotherapy has paved the way for novel therapeutic concepts, including the combination of radiotherapy with immune checkpoint inhibition (e.g. Programmed cell death/ -ligand 1; PD-1/ PD-L1). This approach is of particular interest as it utilizes synergistic effects: While immune checkpoint inhibitors can restore the patients' antitumor immunity through $\mathrm{T}$ cell activation, radiotherapy may further boost immune-mediated anticancer mechanisms by exposing tumor-associated antigens and by attracting both immunocompetent antigenpresenting cells and tumoricidal effector cells $[6,7]$. Indeed, for patients with unresectable stage III NSCLC, the PACIFIC trial has revealed a profound clinical benefit treatment with the anti-PD-L1 monoclonal antibody durvalumab after chemoradiotherapy with remarkably low toxicities $[8,9]$. As a result, sequential treatment with durvalumab after chemoradiotherapy has become the new standard treatment for locally advanced, unresectable NSCLC. However, about $20 \%$ of patients do not receive chemotherapeutic agents, presumably due to significantly higher rates of age- and comorbidityrelated adverse events (AE) under chemoradiotherapy [5, 10]. Thus, elderly and frail patients often receive radiotherapy alone, raising the serious concern of undertreatment and the need for new therapeutic concepts $[4,5]$.

Considering the immune-promoting effects of radiotherapy, a combination with durvalumab therapy may improve response rates in these potentially undertreated patients. Moreover, if applied early, concomitant local radiotherapy with systemic immunotherapy may particularly increase control of distant micrometastases. Preclinical data suggest that irradiation-induced immunogenicity can even be further increased if applied in a hypofractionated setting with single doses $\geq 3$ Gray (Gy), in line with a radiation dose-dependent abscopal effect [11-14]. While a hypofractionated radiation schedule is also considerably shorter and more convenient for the patient, safety of concurrent immunoradiotherapy is a concern, as both therapy modalities may cause severe pneumonitis.

In this prospective phase II clinical trial, we therefore aim to determine feasibility and treatment efficacy of durvalumab treatment combined with thoracic radiotherapy (TRT) in previously untreated NSCLC stage III patients unable to receive radiochemotherapy. Striving to develop a combination treatment strategy that, if proven safe and efficient, can be quickly made available to patients, a two-trials-in-one design was chosen that combines durvalumab with either conventionally fractionated (CON-group) or hypofractionated thoracic radiotherapy (HYPO-group). This study not only aims to increase the efficacy of radiotherapy by utilizing the immune-sensitizing effects elicited by PD-L1 inhibition, but will also provide biomaterials that will be analyzed with respect to immunological mechanisms responsible for checkpoint inhibitor efficacy and immune-promoting effects of radiotherapy as well as potential biomarkers.

\section{Methods/design Study design}

The TRADE-hypo trial is a prospective, randomized, open-label, multicenter, phase II trial with a safety stopand-go lead-in phase (Fig. 1). During the lead-in phase, patients in the HYPO-group, who will receive durvalumab in combination with hypofractionated thoracic radiotherapy, will be closely evaluated with regard to toxicity (defined as pneumonitis $\geq$ grade 3 within 8 


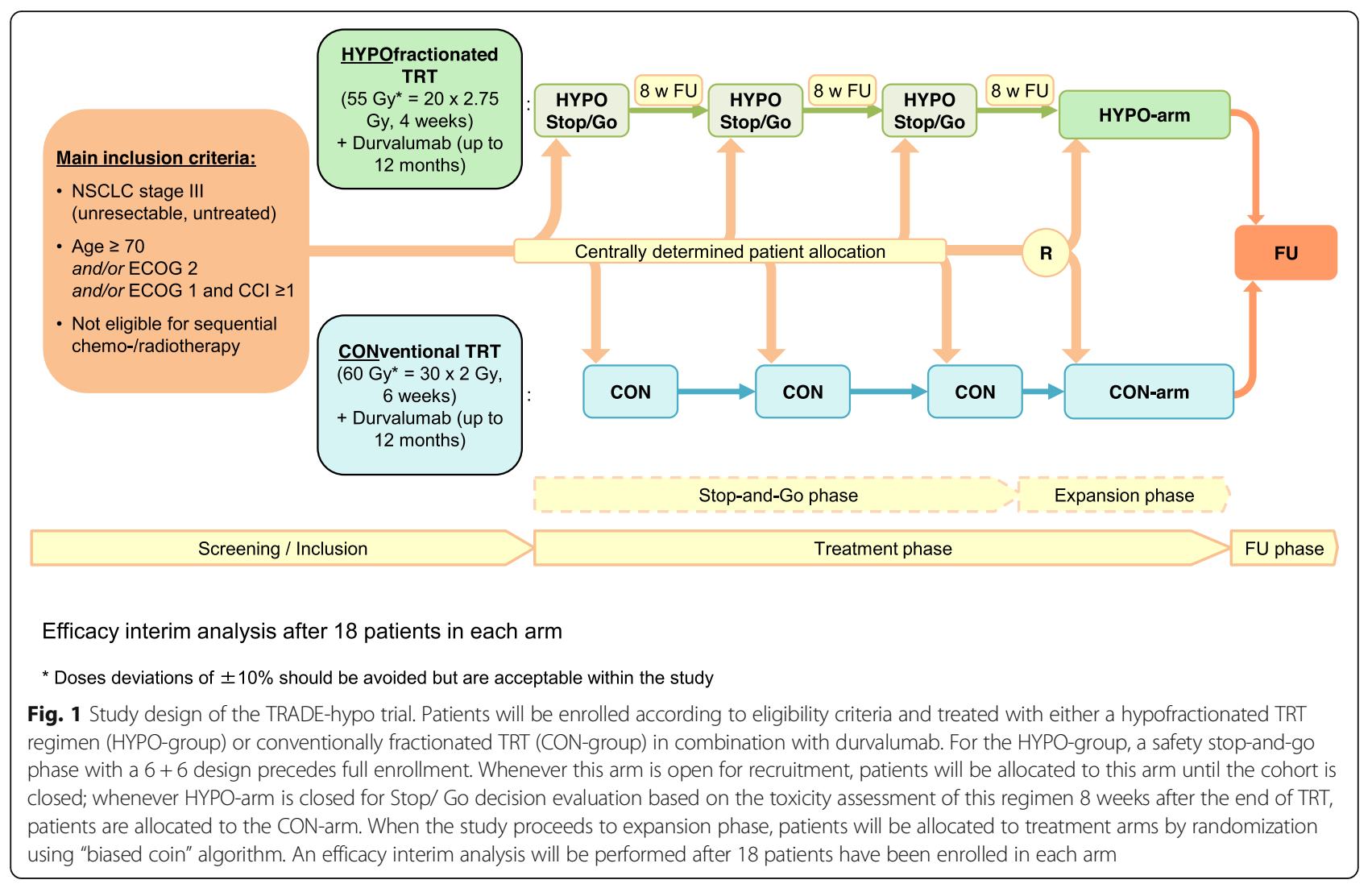

weeks after radiotherapy) in small cohorts $(n=6)$ before proceeding with full enrollment into this arm (Fig. 2).

\section{Study setting}

The TRADE-hypo trial will recruit patients from 17 participating centers across Germany over a period of 20 months. Start of recruitment was planned for April 2020, but was delayed to May 2020 due to the Covid-19 pandemic. A full list of sites can be obtained at clinicaltrials.gov (NCT04351256).

\section{Study objectives}

The primary objective of this study is to evaluate safety and tolerability of conventionally fractionated (CONgroup) and hypofractionated (HYPO-group) TRT in combination with durvalumab in patients with unresectable stage III NSCLC unfit for chemotherapy. Moreover, efficacies of the two modes of radiotherapy will be evaluated with respect to response rates. Further parameters will be determined in order to assess efficacy, safety, and quality of life $(\mathrm{QoL})$ in both treatment arms by recording incidence and severity of adverse events (AEs) as well as specific laboratory abnormalities.

Exploratory endpoints include assessment of vulnerability and analyses of tumor tissue, blood, and stool samples that are collected during the clinical trial with respect to treatment-induced changes and immunerelated biomarkers.

\section{Characteristics of participants}

A total of 88 patients will be included into this study. Patients potentially eligible for trial inclusion will be approached and asked to participate as they present in the clinic. Before a patient's participation in the clinical study, the investigator must obtain written informed consent.

Each participant must be eligible regarding all inclusion and exclusion criteria set for this trial (Table 1). Key inclusion criteria include diagnosis of unresectable stage III NSCLC and non-feasibility of sequential chemoradiotherapy due to increased (oxygen-independent) vulnerability as reflected by fulfilling at least one of the following criteria: (i) Performance status 2 (Eastern Cooperative Oncology Group [ECOG] scale), (ii) ECOG 1 and Charlson comorbidity index $(\mathrm{CCI}) \geq 1$, or (iii) age $\geq$ 70. Moreover, participants must have at least one measurable site of disease as defined by RECIST 1.1, as well as adequate bone marrow, hepatic and renal function. Patients having received prior immunotherapy, other investigational agents or thoracic radiotherapy within the past 5 years will be excluded from the study. Additionally, participants must not have an active or recent history of a known or suspected autoimmune disease or 


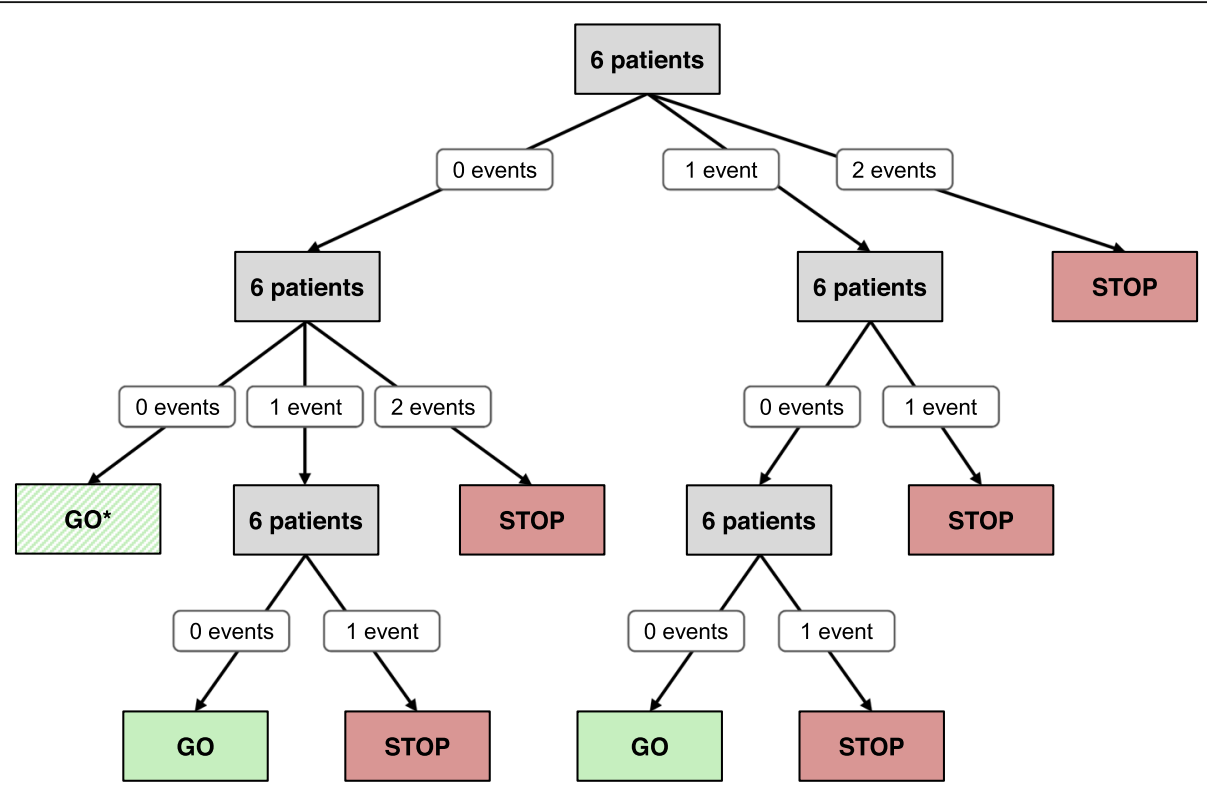

Fig. 2 Cohort design of the safety stop-and-go lead-in phase (HYPO-group). The safety lead-in phase follows a $6+6$ design in order to carefully evaluate the toxicity of the treatment in the HYPO-group with respect of the occurrence of a grade 3/4 pneumonitis ("event") within 8 weeks after the end of TRT. Two events in the first six patients, two events in the first 12, or two events in the first 18 patients will result in termination of the HYPO-group ("Stop"). If no event is observed within the first two safety cohorts, i.e. the first 12 patients, the HYPO-arm will be opened for full enrollment with close toxicity assessment with respect to pneumonitis grade $3 / 4$, and terminated as soon as two events are reported within the subsequent six patients ("Go*"). Full enrollment in the HYPO-arm will only take place if the criteria for the non-toxicity scenario are met, i.e. $\leq$ 1 event in $n=18$ patients ("Go")

any other medical condition conflicting with the study interventions, and not have used immunosuppressive medication. For a full list of the inclusion and exclusion criteria see Table 1 .

\section{Treatment}

\section{Durvalumab}

Patients will be enrolled based on the in-/ exclusion criteria. Two treatment groups will be evaluated: One group will receive durvalumab immunotherapy combined with conventionally fractionated TRT (CONgroup) and the other one with hypofractionated TRT (HYPO-group). In both groups, durvalumab will be administered intravenously at a fixed dose of $1500 \mathrm{mg}$ on day 1 and every 4 weeks thereafter for a maximum of 12 months (maximum 13 doses, last dose at week 49) until confirmed disease progression, inacceptable toxicity, withdrawal of consent or end of the study (Fig. 1 and Table 2).

\section{Radiotherapy}

All patients are subjected to preparation of individual positioning devices and CT-based planning. Motion management may comprise either 4D-CT or midbreathing CT image acquisition. Further imaging modalities, such as FDG-PET/CT, may be used when deemed necessary. Gross tumor volumes (GTV) will be contoured and expanded by adequate clinical (CTV) and planning (PTV) safety margins in order to account for subclinical disease and positioning errors. No elective nodal irradiation will be performed. As for organs at risk, both lungs, spinal cord, heart and esophagus must be contoured. In the HYPO-arm, no more than $30 \%$ of "both lungs minus GTV" should receive > $20 \mathrm{~Gy}$; in the CONarm, no more than $35 \%$ of "both lungs minus GTV" should receive $>20 \mathrm{~Gy}$.

In the HYPO-arm, 20 fractions of 2.75 Gy will be administered (total dose $55 \mathrm{~Gy}$, corresponding to 70 GyBED, $\alpha / \beta=10$ ). In the CON-arm, 30 fractions of $2 \mathrm{~Gy}$ will be administered (total dose $60 \mathrm{~Gy}$, corresponding to 72 GyBED, $\alpha / \beta=10$ ). Dose deviations of $\pm 10 \%$ are acceptable, when clinically indicated. Radiotherapy is scheduled to start within $72 \mathrm{~h}$ after the first administration of durvalumab. Dose prescription will follow international reports (ICRU 50, 62 and 83). Both 3Dconformal and modulated photon radiation techniques, such as IMRT and VMAT/RapdArc, are acceptable. All participating institution are encouraged to perform regular, if no daily, positioning control using either portal imaging or on-board-CT devices.

\section{Study procedures}

In order to minimize the risk exposure of patients when subjected to the hypofractionated radiation regimen, a 
Table 1 Complete list of inclusion and exclusion criteria

\section{Inclusion criteria}

- Fully-informed written consent and locally required authorization obtained from the patient/ legal representative prior to performing any protocolrelated procedures, including screening evaluations.

- Age $\geq 18$ years.

- Histologically documented diagnosis of unresectable stage III NSCLC.

- Non-feasibility of sequential chemo-/radiotherapy as determined by the site's multi-disciplinary tumor board; if there is no tumor board, then this decision will be made by the investigator in consultation with a radiation oncologist, if the investigator is not a radiation oncologist; or by the investigator in consultation with an oncologist, if the investigator is not an oncologist.

- Fulfills at least one of the following criteria:

$\circ$ ECOG 2

o ECOG 1 and $\mathrm{CCl} \geq 1$

$\circ$ Age $\geq 70$ years

- Must have a life expectancy of at least 12 weeks.

- FEV $1 \geq 40 \%$ of predicted

- $\mathrm{DLCO} \geq 40 \%$ of predicted

- FVC or VC $\geq 70 \%$ of predicted

- At least one measurable site of disease as defined by RECIST 1.1 criteria.

- Adequate bone marrow, renal, and hepatic function

- Female patients with reproductive potential must have a negative urine or serum pregnancy test within 7 days prior to start of trial.

- Evidence of post-menopausal status or negative urinary or serum pregnancy test for female pre-menopausal patients.

- The patient is willing and able to comply with the protocol for the duration of the study, including hospital visits for treatment and scheduled follow-up visits and examinations.

\section{Exclusion criteria}

- Concurrent enrollment in another clinical study or enrollment within 21 days prior to first dose of treatment, unless it is an observational (noninterventional) clinical study, or during the follow-up period of an interventional study.

- Prior immunotherapy or use of other investigational agents.

- History or current radiology suggestive of interstitial lung disease.

- Oxygen-dependent medical condition.

- Any concurrent chemotherapy, investigational product (IP), biologic, or hormonal therapy for cancer treatment.

- Prior thoracic radiotherapy within the past 5 years before the first dose of study drug.

- Major surgery within 4 weeks prior to enrollment into the study; patients must have recovered from effects of any major surgery. Local non-major surgery for palliative intent is acceptable.

- Active or prior documented autoimmune or inflammatory disorders, with the following exceptions: Patients with vitiligo or alopecia, patients with hypothyroidism stable on hormone replacement, or any chronic skin condition that does not require systemic therapy. Patients without active disease in the last 5 years may be included but only after consultation with the study physician.

- Active, uncontrolled inflammatory bowel disease. Patients in stable remission for more than 1 year may be included.

- Uncontrolled intercurrent illness, including but not limited to, ongoing or active infection, symptomatic congestive heart failure, uncontrolled hypertension, unstable angina pectoris, uncontrolled cardiac arrhythmia, interstitial lung disease, gastrointestinal conditions associated with diarrhea, or psychiatric illness/social situations that would limit compliance with study requirement, substantially increase risk of incurring AEs or compromise the ability of the patient to give written informed consent.

- History of another primary malignancy except for a malignancy that has been treated with curative intent and was not active for $\geq 5$ years before the first dose of IP and of low potential risk for recurrence or adequately treated non-melanoma skin cancer or lentigo maligna without evidence of disease or adequately treated carcinoma in situ without evidence of disease

- History of leptomeningeal carcinomatosis

- History of active primary immunodeficiency

- History of allogenic organ or tissue transplantation.

- Clinical diagnosis of active tuberculosis.

- Positive testing for hepatitis B virus surface antigen or hepatitis C virus RNA indicating acute or chronic infection or for human immunodeficiency virus.

- Current or prior use of immunosuppressive medication within 14 days before the first dose of durvalumab. The following are exceptions to this criterion: Intranasal, inhaled, topical steroids, or local steroid injections; systemic corticosteroids at physiologic doses not to exceed $10 \mathrm{mg} /$ day of prednisone or its equivalent; steroids as premedication for hypersensitivity reactions

- Receipt of live attenuated vaccine within 30 days prior to the first dose of IP.

- Female patients who are pregnant or breastfeeding or male or female patients of reproductive potential who are not willing to employ effective birth control.

- Known allergy or hypersensitivity to any of the IPs or any of the constituents of the product.

- Any co-existing medical condition that in the investigator's judgement will substantially increase the risk associated with the patient's participation in the study.

- Patient who has been incarcerated or involuntarily institutionalized by court order or by the authorities $\S 40$ Abs. 1 S. 3 Nr. 4 AMG.

- Patients who are unable to consent because they do not understand the nature, significance and implications of the clinical trial and therefore cannot form a rational intention in the light of the facts [§ 40 Abs. 1S. 3 Nr. 3a AMG].

safety lead-in phase with stop-and-go design will precede full enrollment into the HYPO-group (Fig. 2). Toxicity will be evaluated with a $6+6$ design that is based on the statistical assumption that $\leq 1$ event in $n=18$ patients conforms to a non-toxicity scenario, with "event" being defined as the occurrence of pneumonitis grade $\geq 3$ within 8 weeks after end of TRT. Consequently, two events in the first six patients or two events in the first 
Table 2 Schedule of assessments

\begin{tabular}{|c|c|c|c|c|c|c|c|c|c|c|}
\hline \multirow[t]{2}{*}{ Procedure / Point in time } & \multirow{2}{*}{$\begin{array}{l}\text { Screening } \\
\text { Screening }\end{array}$} & \multicolumn{6}{|c|}{ Treatment Cycles (Q4W) } & \multirow{2}{*}{$\begin{array}{l}\text { EOT } \\
\text { EOT }\end{array}$} & \multicolumn{2}{|c|}{ Post-Treatment } \\
\hline & & $\overline{C 1 D 1}$ & C1D4 & C2D1 & C3D1 & C4D1 & C5D1-C13D1 & & Safety FU & $\mathrm{FU}(\mathrm{Q} 12 \mathrm{~W})$ \\
\hline $\begin{array}{l}\text { Informed Consent, eligibility criteria, demographics, } \\
\text { medical and history }\end{array}$ & $x$ & & & & & & & & & \\
\hline Allocation/ Randomization & $x$ & & & & & & & & & \\
\hline Prior and Concomitant Medication Review & $x$ & $x$ & & $x$ & $x$ & $x$ & $x$ & & & \\
\hline Durvalumab administration & & $x$ & & $x$ & $x$ & $x$ & $x$ & & & \\
\hline Radiotherapy $\left(\mathrm{CON}^{\mathrm{a}}\right.$ or $\left.\mathrm{HYPO}^{\mathrm{b}}\right)$ & & & $x$ & & & & & & & \\
\hline AEs & $x$ & $x$ & & $x$ & $x$ & $x$ & $x$ & $x$ & $x$ & $x$ \\
\hline Full Physical Examination & $x$ & & & & & & & $x$ & & \\
\hline Directed Physical Examination & & $x$ & & $x$ & $x$ & $x$ & $x$ & & $x$ & \\
\hline Vital Signs, $\mathrm{O}_{2}$ Saturation, and Weight & $x$ & $x$ & & $x$ & $x$ & $x$ & $x$ & $x$ & $x$ & \\
\hline Pulmonary function tests & $x$ & & & $(X)^{c}$ & $(X)^{c}$ & togethe & ar with staging & $x$ & $x$ & \\
\hline 12-lead ECG & $x$ & Whene & ever clir & ically ir & dicated & & & & & \\
\hline ECOG Performance Status & $x$ & $x$ & & $x$ & $x$ & $x$ & & $x$ & & \\
\hline $\begin{array}{l}\text { Pregnancy Test, CBC with Differential, Serum Chemistry } \\
\text { Panel, Thyroid function test }\end{array}$ & $x$ & $x$ & & $x$ & $x$ & $x$ & $x$ & $x$ & & \\
\hline $\mathrm{HBV} / \mathrm{HCV}$ & $x$ & & & & & & & & & \\
\hline Urinanalysis & $x$ & Whene & ever clir & ically ir & dicated & & & & & \\
\hline Tumor Imaging & $x$ & & & $(X)^{d}$ & $(X)^{d}$ & $x^{e}$ & Q8We & $(X)^{f}$ & & $x^{g}$ \\
\hline FACT-L and G8 screening questionnaire & $x$ & $x$ & & $x$ & $x$ & togethe & with staging & $x$ & $x$ & $(X)^{h}$ \\
\hline Tissue & $x$ & Option & nal: Re-E & jiopsy a & $t$ time $o$ & f progre & ession & & & \\
\hline Blood and stool & $X^{i}$ & $X^{i}$ & & $x$ & & $x$ & & $x$ & & \\
\hline
\end{tabular}

${ }^{a} \mathrm{CON}$-group radiation scheme: Patients receive conventional fractions of $30 \times 2$ Gy ( $60 \mathrm{~Gy}$ ) within 6 weeks of TRT to be started within $72 \mathrm{~h}$ after start of durvalumab treatment

${ }^{b}$ HYPO-group radiation scheme: Patients receive hypofractionated thoracic radiotherapy consisting of $20 \times 2,75$ Gy ( 55 Gy) within 4 weeks of TRT to be started within $72 \mathrm{~h}$ after start of durvalumab treatment

'To be performed on C2D1 and C3D1 if in accordance with local standard

${ }^{d}$ Chest X-ray to be performed on cycles 2 and 3 if in accordance with local standard

${ }^{\mathrm{e}}$ First on-study CT imaging to be performed 12 weeks after first durvalumab administration. Further on-study imaging to be performed Q8W ( 56 days \pm 7 days)

fOnly applicable if EOT not according to already detected disease progression

In patients with EOT not due to disease progression tumor imaging will be performed until the start of a new anticancer treatment, disease progression, death, withdrawal of consent, or the end of the study

${ }^{\mathrm{h}}$ Questionnaires will be collected until disease progression only and may be collected by telephone calls

'Biomarker sample to be taken prior to first study drug medication either during screening or C1D1 visit

12 or two events in the first 18 patients will result in termination of the HYPO-group (Fig. 2).

During this safety stop-and-go phase, patients will be allocated to treatment arms as follows: While the HYPO-arm is recruiting, patients will exclusively enter this treatment group. During safety evaluation of the six patients of a HYPO-cohort (Stop/ Go decision), patients will be allocated to the CON-group only. If safety criteria in the HYPO-cohort are met, the HYPO-arm will be reopened to continue toxicity assessment, and patients will solely be allocated to this arm (Fig. 1). In order to avoid any bias, patients will be allocated centrally by the study management during this phase. If the non-toxicity criteria in the safety cohorts are met, the study will proceed to the expansion phase, and remaining patients will be randomized into the two treatment arms using an interactive web response system integrated in the electronic case report form (eCRF). A possibly uneven distribution of patients between the treatment groups at this stage will be compensated by a randomization strategy based on the "biased coin" method $[15,16]$. In the randomization phase, patients will be stratified according to tumor stage (stage IIIA vs. stage IIIB/IIIC).

In total, 44 patients will be enrolled per group. After $n=18$ patients have been enrolled to the HYPO- or CON-treatment arm, respectively, an interim efficacy analysis for the respective arm will be conducted based on the objective response rate (ORR) at 12 weeks after first durvalumab administration. In case of insufficient efficacy of one of the arms (i.e., the number of patients who have achieved a response is eight out of 18 or lower) this treatment arm will be terminated. Recruitment will be halted until results of the interim efficacy analysis are available. 
Tumor response will be assessed according to RECIST 1.1 by CT and/ or MRI scans at baseline, 12 weeks after durvalumab initiation and then every 8 weeks. Safety measures will include physical examinations, performance status (ECOG), clinical laboratory profiles and continuous assessments of AEs. An overview of all study procedures is presented in Table 2.

An Independent Safety Monitoring Board (ISMB) will ensure the continued safety of participants throughout the trial. Data management and data quality assurance will be conducted following the Standard Operational Procedures of the Institute of Clinical Cancer Research IKF at Northwest Hospital GmbH (Frankfurt, Germany). An eCRF will be carefully maintained for each participant for data collection, also reporting serious and nonserious AEs following the common criteria for adverse events (CTCAE) version 5.0 throughout the entire trial. After the end of the study, participants will be proactively followed up regarding treatment-related AEs until resolved, returned to baseline or considered irreversible, until lost to follow-up, or withdrawal of study consent. All subjects will be followed for survival. Patients who decline to return to the site for evaluations will be offered a follow-up (FU) by phone every 3 months as an alternative. At the end of the study treatment, the investigators are responsible for the further treatment of the patient and must ensure that he or she receives appropriate standard of care or other appropriate therapies.

\section{Sampling for translational research}

If patients participate in the translational research program, blood samples will be collected prior to cycles 1,2 and 4 and at the time of disease progression (or end of treatment, EOT) along with stool samples (Table 2). Samples of untreated tumor lesions serving as baseline specimens will be collected as paraffin-embedded tissue. If re-biopsies are taken at the time of progression, samples should also be also submitted for translational research.

\section{Study endpoints}

The primary endpoint of the study will be toxicity, defined by the occurrence of treatment-related pneumonitis grade $\geq 3$. The ORR evaluated 12 weeks after first durvalumab administration (according to RECIST 1.1) is set as the primary efficacy endpoint. Secondary endpoints of the study comprise the occurrence of treatment-related AEs and serious AEs (SAEs), frequency of prespecified abnormal laboratory parameters, progression-free survival (PFS) and duration of clinical benefit, metastasis-free survival, overall survival (OS), and QoL.
Patient vulnerability and its association with survival and outcome will be assessed as an exploratory endpoint. To this end, the G8-screening questionnaire, a simple and fast screening tool for identifying geriatric risk profiles with a strong prognostic value for functional decline and OS in older populations with cancer, will be used [17]. Furthermore, biomaterials will be collected during the trial for correlation analyses on selected molecular parameters and clinical data in order to identify molecular biomarkers predictive for response to therapy. This approach is deemed appropriate to obtain hypothesis-generating data for future research due to the explorative character of this trial.

\section{Statistical analysis \\ Sample size justification}

Safety run-in phase (HYPO-group) With regard to the pneumonitis grade $\geq 3$ rate, this phase is designed to distinguish between a toxicity scenario $(\mathrm{pTox}=0.15)$ and a non-toxicity scenario (pTox $=0.035)$. A sample size of $n=18$ will yield a probability of 0.78 to correctly detect the toxicity scenario, while the non-toxicity scenario will correctly be detected with a probability of 0.87 . These probabilities are based on the decision rule that, if the number of patients with pneumonitis grade $\geq 3$ in this cohort is $\geq 2$, recruitment to the HYPO-group will be terminated.

Interim efficacy analysis regarding ORR and expansion phase An interim efficacy analysis based on the ORR will be conducted after $n=18$ patients in each arm have completed radiotherapy and the 18th patient has undergone first radiographic assessment (at 12 weeks after first durvalumab dose).

Previously, an ORR of $45 \%$ after radiotherapy alone has been reported in a Japanese population of elderly patients with unresectable stage III NSCLC [18]. Based on this and the observation that Asian ethnicity is associated with a favorable prognosis, we assume for the TRADE-hypo trial that an ORR higher than 0.42 in both treatment arms can be demonstrated, i.e. the null hypotheses for arm HYPO and CON are defined as $\mathrm{H}_{0}$ ${ }_{\mathrm{HYPO}}: \pi{ }^{\mathrm{HYPO}} \leq 0.42$ and $\mathrm{H}_{0}{ }^{\mathrm{CON}}$ : $\pi{ }^{\mathrm{CON}} \leq 0.42$, where $\pi$ HYPO and $\pi^{\text {CON }}$ denote the actual ORR in arm HYPO and $\mathrm{CON}$, respectively $[19,20]$. Under the alternative hypothesis, it is assumed that both $\pi^{\mathrm{HYOO}}$ and $\pi \mathrm{CON}$ amount to 0.60. Using an optimal Simon's two-stage design with a one-sided significance level of $\alpha=0.10$ and a power of $1-\beta=0.80$ for each hypothesis test, $n=40$ patients per arm are required, while the interim analysis is conducted after $\mathrm{n}=18$ patients per arm have been recruited to the trial. After successfully passing the safety analysis in the HYPO-group, if among 18 patients in the 
HYPO- or CON-arm, the number of patients who have achieved a response is eight or lower, the respective arm will be closed. Otherwise, an additional number of 22 patients will be enrolled into the respective arm. The null hypothesis of the respective arm can be rejected if at least 21 of all 40 patients per arm achieve a response. Sample size calculation was done using the $\mathrm{R}$ package "OneArmPhaseTwoStudy" [21].

To account for an estimated dropout rate of $10 \%$, four patients will additionally be recruited to each treatment arm. Deviations from planned sample sizes will be handled as described by Englert \& Kieser, allowing strict control of the aspired type I error rate in each arm [22].

Methods of statistical analysis The primary population for evaluating all efficacy endpoints and subject characteristics is defined as all patients enrolled according to initial allocation/randomization (intention-to-treat population, IIT). Secondary efficacy analyses will be carried out on the per-protocol (PP) population. The safety population comprising all patients who received at least one dose of the study medication will be used for all safety endpoints and will be analyzed according to the actual treatment received.

Response rates with confidence intervals $(\mathrm{CI})$ and $p$ values in both study arms will be estimated taking into account the two-stage nature of the design [23, 24]. Secondary endpoints will be evaluated descriptively. All toxicities will be summarized by relative and absolute frequency, and severity grade based on CTCAE V5.0. AE and SAE summary tables will provide the number and percentage of patients with AEs and the 95\% ClopperPearson type CIs. All analyses will be done using SAS version 9.4 (SAS Institute, Cary, NC) or higher.

\section{Trial status}

As of July 15th 2020, eight study sites are initiated. First initiation coincided with the beginning of the Covid-19 pandemic in Germany. Therefore, recruitment of patients was withheld. On May 8th 2020, recruitment was resumed after consultation with the ISMB. The first patient was enrolled on July 13th 2020.

\section{Discussion}

In recent years, the concept of restoring the patients' antitumor immunity by immune checkpoint inhibition has revolutionized cancer therapy, especially in advanced melanoma, renal carcinoma and NSCLC. Immune checkpoint molecules efficiently regulate $\mathrm{T}$ cell activation, and thus enable tumor cells to evade the immune system, for example by hijacking the PD-1/ PD-L1 interaction to downregulate effector T cells $[25,26]$. To date, several human monoclonal antibodies pharmacologically blocking these interactions have been implemented in cancer therapy, such as the anti-PD-1 antibody pembrolizumab that has been approved in combination with chemotherapy for non-squamous NSCLC, irrespective of PD-L1 expression [27, 28].

Several studies have shown that immune checkpoint inhibition and radiotherapy in combination can act synergistically to further boost antineoplastic effects [2932]. Although in a large phase III trial, no benefit of blockade of cytotoxic $\mathrm{T}$ lymphocyte-associated antigen 4 (CTLA-4) after radiotherapy was observed in metastatic prostate cancer [33], clinical trials, such as NICOLAS (NCT02434081) and DETERRED (NCT02525757), investigating concurrent $\mathrm{PD}$-(L)1-directed immunotherapy and chemoradiotherapy in patients with locally advanced lung cancer, have confirmed efficiency at modest toxicity rates, particularly with respect to pneumonitis, which is one of the most threatening complications in NSCLC patients [34-36]. The PACIFIC trial showed an impressive improvement regarding PFS and OS with manageable side effects with durvalumab treatment after photon radiotherapy combined with chemotherapy in stage III, unresectable NSCLC patients, which has resulted in the approval of durvalumab consolidation after chemoradiotherapy $[8,9]$. Safety of durvalumab in combination with concurrent palliative radiotherapy has also been confirmed in a smaller series of heterogeneous metastatic tumor patients [37]. Further, the combination may also prove to be an effective therapeutic concept in other entities, as for example is currently investigated in patients with head-and-neck cancer (NCT03283605) [38].

With regard to elderly and frail patients with unresectable stage III NSCLC unfit for chemotherapy, we hypothesize that durvalumab in combination with mildly hypofractionated TRT is safe and effective, given that feasibility and activity of this regime have been demonstrated in combination with chemotherapy in stage III patients $[39,40]$. To date, no prospective trial has investigated such a therapeutic approach. Taking into account the concern of a cumulative risk for severe pneumonitis from applying both TRT and immunotherapy, the TRADE-hypo trial will investigate two radiation regimens combining durvalumab therapy with either conventionally fractionated or hypofractionated TRT.

The study design includes a safety stop-and-go phase preceding full enrollment to ensure that the hypofractionated treatment regimen can instantly be discontinued if deemed unsafe. Taking this and the interim efficacy analysis into account, this study is designed to efficiently avoid inadequate therapy and unnecessary costs. On the other hand, it may reveal a safe and efficient first-line immunoradiotherapeutic strategy for frail and elderly NSCLC stage III patients unable to receive chemotherapy, and, thus, provide an additional and optimized therapeutic option for this potentially undertreated 
patient cohort. Furthermore, data from this study may guide the design of larger, randomized trials investigating such an approach in this context.

In the accompanying translational research project, the host-tumor biology will be analyzed with a focus on immunological mechanisms responsible for antitumoral effects of both checkpoint inhibitors and radiotherapy, in an attempt to identify biomarkers predictive of therapy response. Eventually, this study may provide valuable data to explore how antitumoral immunological mechanisms may be activated in non-responders, currently representing about half the patients treated with immune-checkpoint inhibitors [27, 28, 41].

\begin{abstract}
Abbreviations
AE: Adverse event; CCl: Charlson comorbidity index; Cl: Confidence interval; CT: Computed tomography; CTCAE: Common Terminology Criteria for Adverse Event; CTLA-4: Cytotoxic T lymphocyte-associated antigen 4; CTV: Clinical target volume; ECG: Electrocardiogram; ECOG: Eastern Cooperative Oncology Group; eCRF: Electronic case report form; EOT: End of treatment; FU: Follow-up; GTV: Gross tumor volume; Gy: Gray; IP: Investigational Product; ISMB: Independent Safety Monitoring Board; ITT: Intent-to-treat; MRI: Magnetic resonance imaging; NSCLC: Non-small cell lung cancer; ORR: Objective response rate; OS: Overall survival; PD1: Programmed cell death 1; PD-L1: Programmed cell death ligand 1; PFS: Progression-free survival; PP: Per-protocol; PTV: Planning target volume; QoL: Quality of life; RECIST 1.1: Response Evaluation Criteria in Solid Tumors,
\end{abstract} version 1.1; SAE: Serious adverse event; TRT: Thoracic radiation therapy

\section{Acknowledgements}

The authors would like to thank the members of the Independent Safety Monitoring Board. This trial is conducted in cooperation with the Young Medical Oncologists Group and the Thoracic Oncology Working Group of the Arbeitsgemeinschaft Internistische Onkologie (AIO) within the German Cancer Society. We acknowledge financial support by the BadenWürttemberg Ministry of Science, Research and the Arts and by the Ruprecht-Karls-University Heidelberg.

\section{Authors' contributions}

FB, IC, MT and SR developed the study idea, wrote the protocol and coordinated funding, regulatory permission and approval processes. FB is lead investigator of this study; SR is deputy lead investigator and will supervise all radiation treatments. JK calculated (bio-) statistical models for sample size, study hypothesis, and endpoint determinations. PC and FB developed biomarker evaluation strategies and MS will coordinate sample collection and processing during this study. LB participated in writing the manuscript. DM is representative of the Institute of Clinical Cancer Research IKF at Northwest Hospital GmbH that is legal sponsor of the study, gave advice on study design and is responsible for study management and logistics. All authors have read and approved the current version of the manuscript.

\section{Funding}

The Institute of Clinical Cancer Research IKF at Northwest Hospital GmbH (Steinbacher Hohl 2-26, D-60488 Frankfurt a.M.) acts as the legal sponsor and finances the trial. Financial support and drug supply is granted by the pharmaceutical company AstraZeneca/ Medlmmune. Open access funding provided by Projekt DEAL.

\section{Availability of data and materials}

Data generated by this study will be available for access from the corresponding author upon reasonable request.

\section{Ethics approval and consent to participate}

Ethical approval by the Ethics Committee of the Medical Faculty of the University of Heidelberg was obtained (AFmu-683/2019 March 18, 2020). Before participation in the clinical study, written informed consent will be obtained from each subject. Furthermore, the DEGRO expert panel reviewed the radiation regimen to rule out that permission of radiation administered would have to be requested from the BfS (Federal office of radiation protection; "Anfrage 167 / 09 July, 2019"). Additionally, the Paul Ehrlich Institute (competent authority for approval of clinical trials using medicinal products for human use in Germany) approved the study (no. 3881/02, March 10, 2020).

\section{Consent for publication}

Not applicable, as no individual patient data are contained in this manuscript.

\section{Competing interests}

The TRADE-hypo trial receives funding from AstraZeneca/ Medlmmune. However, AstraZeneca/ Medlmmune has not been involved in study design, data collection, management, data analysis and interpretation, or in the decision to submit this protocol for publication. All authors declare that they have no competing interests.

\section{Author details}

'Department of Thoracic Oncology, Thoraxklinik at University Hospital of Heidelberg, Röntgenstraße 1, 69126 Heidelberg, Germany. ${ }^{2}$ Translational Lung Research Center Heidelberg TLRCH, Member of the German Center for Lung Research DZL, Im Neuenheimer Feld 156, 69120 Heidelberg, Germany. ${ }^{3}$ Institute of Medical Biometry and Informatics, University Hospital of Heidelberg, Im Neuenheimer Feld 130.3, 69120 Heidelberg, Germany. ${ }^{4}$ Thoraxklinik at University Hospital of Heidelberg, Translational Research Unit (STF), Röntgenstraße 1, 69126 Heidelberg, Germany. Institute of Clinical Cancer Research IKF GmbH at Northwest Hospital, Steinbacher Hohl 2-26, 60488 Frankfurt am Main, Germany. ${ }^{6}$ Department of Radiation Oncology, University Medical Center Göttingen, Robert-Koch-Str. 40, 37075 Göttingen, Germany.

Received: 16 July 2020 Accepted: 5 August 2020

Published online: 26 August 2020

\section{References}

1. Planchard D, Popat S, Kerr K, Novello S, Smit EF, Faivre-Finn C, Mok TS, Reck $M$, Van Schil PE, Hellmann MD, et al. Metastatic non-small cell lung cancer: ESMO Clinical Practice Guidelines for diagnosis, treatment and follow-up. Ann Oncol. 2018;29(Suppl 4):iv192-237.

2. Siegel RL, Miller KD, Jemal A. Cancer statistics, 2020. CA Cancer J Clin. 2020; 70(1):7-30.

3. Howlader NNAKM, et al. SEER Cancer statistics review, 1975-2010. Bethesda: National Cancer Institute; 2013.

4. Miller ED, Fisher JL, Haglund KE, Grecula JC, Xu-Welliver M, Bertino EM, He K, Shields PG, Carbone DP, Williams TM, et al. Identifying patterns of care for elderly patients with non-surgically treated stage III non-small cell lung cancer: an analysis of the national cancer database. Radiat Oncol. 2018;13(1):196.

5. Driessen EJM, Schulkes KJG, Dingemans AC, van Loon JGM, Hamaker ME, Aarts MJ, Janssen-Heijnen MLG. Patterns of treatment and survival among older patients with stage III non-small cell lung cancer. Lung Cancer. 2018; 116:55-61.

6. Formenti SC, Demaria S. Systemic effects of local radiotherapy. Lancet Oncol. 2009;10(7):718-26.

7. Ko EC, Raben D, Formenti SC. The integration of radiotherapy with immunotherapy for the treatment of non-small cell lung Cancer. Clin Cancer Res. 2018;24(23):5792-806.

8. Antonia SJ, Villegas A, Daniel D, Vicente D, Murakami S, Hui R, Yokoi T, Chiappori A, Lee KH, de Wit M, et al. Durvalumab after Chemoradiotherapy in stage III non-small-cell lung Cancer. N Engl J Med. 2017;377(20):1919-29.

9. Antonia SJ, Villegas A, Daniel D, Vicente D, Murakami S, Hui R, Kurata T, Chiappori A, Lee KH, de Wit M, et al. Overall survival with Durvalumab after Chemoradiotherapy in stage III NSCLC. N Engl J Med. 2018;379(24):2342-50,

10. Stinchcombe TE, Zhang Y, Vokes EE, Schiller JH, Bradley JD, Kelly K, Curran WJ Jr, Schild SE, Movsas B, Clamon G, et al. Pooled analysis of individual patient data on concurrent Chemoradiotherapy for stage III non-small-cell lung Cancer in elderly patients compared with younger patients who participated in US National Cancer Institute cooperative group studies. $J$ Clin Oncol. 2017;35(25):2885-92. 
11. Schaue D, Ratikan JA, Iwamoto KS, McBride WH. Maximizing tumor immunity with fractionated radiation. Int J Radiat Oncol Biol Phys. 2012; 83(4):1306-10.

12. Dewan MZ, Galloway AE, Kawashima N, Dewyngaert JK, Babb JS, Formenti SC, Demaria S. Fractionated but not single-dose radiotherapy induces an immune-mediated abscopal effect when combined with anti-CTLA-4 antibody. Clin Cancer Res. 2009;15(17):5379-88.

13. Lugade AA, Moran JP, Gerber SA, Rose RC, Frelinger JG, Lord EM. Local radiation therapy of B16 melanoma tumors increases the generation of tumor antigen-specific effector cells that traffic to the tumor. J Immunol. 2005;174(12):7516-23.

14. Marconi R, Strolin S, Bossi G, Strigari L. A meta-analysis of the abscopal effect in preclinical models: is the biologically effective dose a relevant physical trigger? PLoS One. 2017;12(2):e0171559.

15. Smith R. Sequential treatment allocation using biased coin designs. J R Stat Soc Ser B Methodol. 1984;46(3):519-43.

16. Frane J. A method of biased coin randomization, its implementation, and its validation. Drug Information J. 1998:32(2):423-32.

17. Kenis C, Decoster L, Van Puyvelde K, De Greve J, Conings G, Milisen K Flamaing J, Lobelle JP, Wildiers $\mathrm{H}$. Performance of two geriatric screening tools in older patients with cancer. J Clin Oncol. 2014;32(1):19-26.

18. Atagi S, Kawahara M, Yokoyama A, Okamoto H, Yamamoto N, Ohe Y, Sawa T, Ishikura S, Shibata T, Fukuda H, et al. Thoracic radiotherapy with or without daily low-dose carboplatin in elderly patients with non-small-cell lung cancer: a randomised, controlled, phase 3 trial by the Japan clinical oncology group (JCOG0301). Lancet Oncol. 2012;13(7):671-8.

19. Ou SH, Ziogas A, Zell JA. Asian ethnicity is a favorable prognostic factor for overall survival in non-small cell lung cancer (NSCLC) and is independent of smoking status. J Thorac Oncol. 2009;4(9):1083-93.

20. Zhou W, Christiani DC. East meets west: ethnic differences in epidemiology and clinical behaviors of lung cancer between east Asians and Caucasians. Chin J Cancer. 2011;30(5):287-92.

21. Kieser MWM, Englert S, Kunz CU, Rauch G. OneArmPhaseTwoStudy: an R package for planning, conducting, and analysing single-arm phase II studies. J Stat Soft. 2017;81:1-28.

22. Englert S, Kieser M. Methods for proper handling of overrunning and underrunning in phase II designs for oncology trials. Stat Med. 2015;34(13): 2128-37.

23. Kunzmann K, Kieser M. Point estimation and p-values in phase II adaptive two-stage designs with a binary endpoint. Stat Med. 2017;36(6):971-84.

24. Kunzmann K, Kieser M. Test-compatible confidence intervals for adaptive two-stage single-arm designs with binary endpoint. Biom J. 2018;60(1):196-206.

25. Dong H, Strome SE, Salomao DR, Tamura H, Hirano F, Flies DB, Roche PC, Lu J, Zhu G, Tamada K, et al. Tumor-associated B7-H1 promotes T-cell apoptosis: a potential mechanism of immune evasion. Nat Med. 2002:8(8): 793-800.

26. Iwai $Y$, Ishida M, Tanaka Y, Okazaki T, Honjo T, Minato N. Involvement of PDL1 on tumor cells in the escape from host immune system and tumor immunotherapy by PD-L1 blockade. Proc Natl Acad Sci U S A. 2002;99(19): 12293-7.

27. Reck M, Rodriguez-Abreu D, Robinson AG, Hui R, Csoszi T, Fulop A, Gottfried M, Peled N, Tafreshi A, Cuffe S, et al. Pembrolizumab versus chemotherapy for PD-L1-positive non-small-cell lung Cancer. N Engl J Med. 2016;375(19): 1823-33.

28. Gandhi L, Rodriguez-Abreu D, Gadgeel S, Esteban E, Felip E, De Angelis F, Domine M, Clingan P, Hochmair MJ, Powell SF, et al. Pembrolizumab plus chemotherapy in metastatic non-small-cell lung Cancer. N Engl J Med. 2018; 378(22):2078-92.

29. Shaverdian N, Lisberg AE, Bornazyan K, Veruttipong D, Goldman JW, Formenti SC, Garon EB, Lee P. Previous radiotherapy and the clinical activity and toxicity of pembrolizumab in the treatment of non-small-cell lung cancer: a secondary analysis of the KEYNOTE-001 phase 1 trial. Lancet Oncol. 2017;18(7):895-903.

30. Dovedi SJ, Adlard AL, Lipowska-Bhalla G, McKenna C, Jones S, Cheadle EJ, Stratford IJ, Poon E, Morrow M, Stewart R, et al. Acquired resistance to fractionated radiotherapy can be overcome by concurrent PD-L1 blockade. Cancer Res. 2014;74(19):5458-68.

31. Golden EB, Chhabra A, Chachoua A, Adams S, Donach M, Fenton-Kerimian M, Friedman K, Ponzo F, Babb JS, Goldberg J, et al. Local radiotherapy and granulocyte-macrophage colony-stimulating factor to generate abscopal responses in patients with metastatic solid tumours: a proof-of-principle trial. Lancet Oncol. 2015;16(7):795-803.

32. Ngwa W, Irabor OC, Schoenfeld JD, Hesser J, Demaria S, Formenti SC. Using immunotherapy to boost the abscopal effect. Nat Rev Cancer. 2018;18(5): 313-22.

33. Kwon ED, Drake CG, Scher HI, Fizazi K, Bossi A, van den Eertwegh AJ, Krainer M, Houede N, Santos R, Mahammedi H, et al. Ipilimumab versus placebo after radiotherapy in patients with metastatic castration-resistant prostate cancer that had progressed after docetaxel chemotherapy (CA184-043): a multicentre, randomised, double-blind, phase 3 trial. Lancet Oncol. 2014; 15(7):700-12.

34. Peters S, Felip E, Dafni U, Belka C, Guckenberger M, Irigoyen A, Nadal E, Becker A, Vees H, Pless M, et al. Safety evaluation of nivolumab added concurrently to radiotherapy in a standard first line chemo-radiotherapy regimen in stage III non-small cell lung cancer-the ETOP NICOLAS trial. Lung Cancer. 2019;133:83-7.

35. Lin SH, Lin Y, Yao L, Kalhor N, Carter BW, Altan M, Blumenschein G, Byers LA Fossella F, Gibbons DL, et al. Phase II trial of concurrent Atezolizumab with Chemoradiation for Unresectable NSCLC. J Thorac Oncol. 2020;15(2):248-57.

36. Belluomini L, Fiorica F, Frassoldati A. Immune checkpoint inhibitors and radiotherapy in NSCLC patients: not just a fluke. Oncol Ther. 2019;7(1):83-91.

37. Levy A, Massard C, Soria JC, Deutsch E. Concurrent irradiation with the antiprogrammed cell death ligand-1 immune checkpoint blocker durvalumab: single Centre subset analysis from a phase 1/2 trial. Eur J Cancer. 2016;68: 156-62.

38. Bahig H, Aubin F, Stagg J, Gologan O, Ballivy O, Bissada E, Nguyen-Tan FP, Soulieres D, Guertin L, Filion E, et al. Phase I/II trial of Durvalumab plus Tremelimumab and stereotactic body radiotherapy for metastatic head and neck carcinoma. BMC Cancer. 2019;19(1):68

39. Maguire J, Khan I, McMenemin R, O'Rourke N, McNee S, Kelly V, Peedell C, Snee M. SOCCAR: a randomised phase II trial comparing sequential versus concurrent chemotherapy and radical hypofractionated radiotherapy in patients with inoperable stage III non-small cell lung Cancer and good performance status. Eur J Cancer. 2014;50(17):2939-49.

40. Iabal MS, Vashisht G, Mulvenna P, McDonald F, Turnbull H, Atherton P. Bradshaw A, Simmons T, Kovarik J, Singhal S, et al. Hypofractionated concurrent chemoradiation in non-small cell lung cancer (NSCLC): efficacy and toxicity of the SOCCAR trial regime in real world practice. Lung Cancer. 2018;115:S64.

41. Hellmann MD, Rizvi NA, Goldman JW, Gettinger SN, Borghaei H, Brahmer JR, Ready NE, Gerber DE, Chow LQ, Juergens RA, et al. Nivolumab plus ipilimumab as first-line treatment for advanced non-small-cell lung cancer (CheckMate 012): results of an open-label, phase 1, multicohort study. Lancet Oncol. 2017;18(1):31-41.

\section{Publisher's Note}

Springer Nature remains neutral with regard to jurisdictional claims in published maps and institutional affiliations.
Ready to submit your research? Choose BMC and benefit from:

- fast, convenient online submission

- thorough peer review by experienced researchers in your field

- rapid publication on acceptance

- support for research data, including large and complex data types

- gold Open Access which fosters wider collaboration and increased citations

- maximum visibility for your research: over $100 \mathrm{M}$ website views per year

At $\mathrm{BMC}$, research is always in progress.

Learn more biomedcentral.com/submission 